\title{
Transport by pulsatile flow in a branching network of cerebral vasculature
}

\author{
I. D. Šutalo ${ }^{1,2}$, A. V. Bui ${ }^{3}$, K. Liffman ${ }^{1,2}$ \& R. Manasseh ${ }^{4,5}$ \\ ${ }^{1}$ Materials Science and Engineering, Commonwealth Scientific and \\ Industrial Research Organisation (CSIRO), Australia \\ ${ }^{2}$ Curtin Health Innovation Research Institute, Curtin University, Australia \\ ${ }^{3}$ Idaho National Laboratory, USA \\ ${ }^{4}$ Faculty of Engineering and Industrial Sciences, \\ Swinburne University of Technology, Australia \\ ${ }^{5}$ Mechanical Engineering, The University of Melbourne, Australia
}

\begin{abstract}
The supply of oxygen and glucose by blood flow is vital to the normal function of the brain and the deficit of either of these metabolism elements can cause severe degradation of the brain functionality. The transport of materials in the complex multi-branching structure of the cerebral vasculature is investigated to predict brain oxygenation under normal conditions.

A mathematical model of material transport due to pulsatile flow in a complex dichotomous branching tree network was developed which incorporated material-geometry interaction and diffusion across the blood vessel wall. Unlike previous work, this modelling work includes the full network structure and incorporates time-dependent flow.

The predicted results indicate some effect of the flow transients on the propagation of the material introduced at the root segment in the vascular network. The effect was more pronounced in the case of constant blood viscosity. The transport model addressed the issue of oxygen transport in the cerebral vascular branching network with the inclusion of red blood cell (RBC) separation at bifurcation points. The predicted results indicate the significance of the vascular network geometry and RBC-bifurcation point interaction in defining the homogeneity of flow and oxygenation by the fractal vasculature. The simulations are found to be able to provide insights into the transport of materials by the blood circulation in the cerebral vasculature and the various factors which
\end{abstract}


may affect the process. The separation of RBCs at branching points has a profound effect on the haemoglobin transport and, consequently, oxygen distribution in the vascular branching network.

Keywords: cerebral vasculature, branching tree, pulsatile flow, red blood cells, brain oxygenation.

\section{Introduction}

Diffusion and convection of materials in the complex network of bloodsupplying vessels have been important issues in pharmacokinetics, toxicokinetics, and medical imaging research. In these studies, heterogeneity of the flow and material distributions and the transit time kinetics are the major investigative issues [1]. Transport of materials by blood flow also is an important subject of physiological and pathological studies, which can elucidate the interdependency of the blood flow and the brain oxygenation and hypoxia [2, 3] and help to reveal the mechanism of embolic stroke $[4,5]$.

There are three major aspects of material transport by blood flow, namely convection by the flow, diffusion across the vessel walls, and consumption in or release from the tissue. The flows in microvasculatures have been numerically analysed in the past [6-9]. However, most of these works considered the networks of small arteries as binary asymmetric structured trees attached to the terminals of large arteries [6,7]. They did not model the detailed structure of the small arterial network and the flow inside it. Mayer [9] and Gabryś et al. [8] modelled the flows in different bifurcation levels of more detailed branching networks constructed on the basis of the fractal scaling principle, but the important effect of the flow pulsation (and the arterial vessel compliance) was not considered.

Various factors may affect the diffusion of materials across the blood vessel walls. For instance, the blood-brain barrier is known for having an important restricting effect on the passage of chemical substances and objects from the blood to the brain tissue [10]. Edvinsson et al. [11] described how there is a close relationship between oxygen consumption (metabolism) and blood flow in the brain and how their interdependence has a profound implication both on the normal functioning of central nervous system as well as on the possible pathological progression of various disorders and diseases.

Some numerical studies of material and, in particular, oxygen transports by the blood flow in models of blood vessel networks were considered [2-4, 12-20]. Often, the complex vascular networks were structurally simplified [3, 4, $12,13,16]$ to reduce the difficulty of numerical procedure and/or to have analytical solutions attainable. In most of the above studies, the transient effect of the flow on material transport was ignored.

In this study, a mathematical model of material transport due to pulsatile flow in a complex dichotomous branching tree network was developed which incorporated material-geometry interaction and diffusion across the blood vessel wall. The aim of this study is to investigate the effect of transient flow, RBC separation, oxygen diffusion across wall on the material transport in the Middle 
Cerebral Artery (MCA) cerebral vasculature. Unlike previous work, this modelling work includes the full network structure and incorporates timedependent flow.

\section{Methods}

\subsection{Pulsatile flow inside a fractal branching-tree model of the cerebral vascular system}

In this work, a three-dimensional (3D) branching-tree model of MCA cerebral vasculature has been constructed on the basis of fractal scaling principal using the Constrained Constructive Optimization method (CCO) [21]. A model of pulsatile flow and pressure distribution in a vascular branching network, which takes into consideration the effect of variable blood rheology and blood vessel compliance, has been developed by Bui et al. [19]. They showed that the flow and pressure distributions in a complex vascular branching network can be described by a system of differential algebraic equations (DAEs) representing the mass conservation at the branching points. In this work, the MCA flow rate is $2.78 \mathrm{~mL} / \mathrm{s}$, perfusion volume is $280 \mathrm{~cm}^{3}$ and the total number of segments is 5559. The flow at the simulate nodes are listed in Table 1.

Table 1: $\quad$ Flow at simulation nodes.

\begin{tabular}{|l|c|c|c|}
\hline Node & Flow ratio & Bifurcation level & Strahler order $^{*}$ \\
\hline N1(root) & 1 & 1 & 7 \\
\hline N2 & 0.38 & 7 & 6 \\
\hline N3 & 0.077 & 15 & 5 \\
\hline N4 & 0.024 & 24 & 4 \\
\hline N5 & 0.01 & 31 & 3 \\
\hline
\end{tabular}

${ }^{*}$ The Strahler order defines branch size based on smaller branches in the tree. The Shrahler number is a numerical measure of branch complexity.

\subsection{Material transport inside a vascular branching networks}

Inside a segment of a vascular branching tree, the mass conservation of the material can be written as follows with the diffusion term omitted [5]:

$$
V \frac{d\left(c_{\text {in }}+c_{\text {out }}\right)}{d \mathrm{t}}=\left(Q_{\text {in }}+Q_{\text {out }}\right)\left(c_{\text {in }}-c_{\text {out }}\right)+2 \dot{m}_{c}
$$

where $c_{i}$ is concentration, $V$ is segment volume, $Q$ is mass flow rate and $\dot{m}_{c}$ is the generation or loss of material $i$. Eqn (1) has a form similar to the oxygen transport formulation given by Boas et al. [3]. With the volume flow rate at all branching points defined as described in section 2.1 [19], the above transport equation can be solved for all segments of the network with the concentration at the root of the branching tree prescribed as the boundary condition. 


\subsection{Oxygen transport inside the vascular network and in the surrounding tissues}

An important aspect of the transport in the vascular network is the delivery of oxygen by the blood to the tissue. Oxygen is transported in the blood mainly by haemoglobin $(\mathrm{Hb})$. To a much lesser degree, oxygen is also dissolved in and carried by the blood's plasma. Both of the above transport mechanisms are dependent on the partial pressure of oxygen $p_{\mathrm{O}_{2}}$. The total concentration of oxygen in the blood is therefore defined as:

$$
c=4 c_{H b} S\left(p_{O_{2}}\right)+c_{s}\left(p_{O_{2}}\right) .
$$

The first term in eqn. (2) describes the oxygen transport due to haemoglobin convection with $4 c_{\mathrm{Hb}}$ indicating the concentration of oxygen bound in RBC haemoglobin molecules at saturated state (i.e. each haemoglobin molecule binds with four oxygen molecules) and $S$ being the haemoglobin saturation, which is commonly related to the oxygen partial pressure by Hill's correlation, as follows:

$$
S\left(p_{\mathrm{O}_{2}}\right)=\frac{\left(p_{\mathrm{O}_{2}} / p_{50}\right)^{n}}{1+\left(p_{\mathrm{O}_{2}} / p_{50}\right)^{n}}
$$

where $p_{50}$ is the oxygen partial pressure at $50 \%$ haemoglobin saturation and $n$ is the haemoglobin cooperativity. The dependence of oxygen saturation in haemoglobin on the partial pressure of oxygen in the blood is called the oxygenhaemoglobin dissociation curve, which indicates the haemoglobin oxygen binding characteristics or the haemoglobin's affinity for oxygen. This dependence may change (or shift) due to different factors, such as age, body temperature, or hydrogen ion concentration $(\mathrm{pH})$. An increase of $p_{50}$ indicates the haemoglobin's increased affinity for oxygen. As oxygen must dissolve into plasma before diffusing through the blood vessel wall to the tissue, the oxygenhaemoglobin dissociation curve is an important factor defining the dynamics of oxygen delivery. With the use of Hill's correlation to correlate haemoglobin saturation and oxygen partial pressure, the kinetics of the oxygen-haemoglobin dissociation reaction is not considered.

The second term in eqn. (2) representing the oxygen concentration in plasma is determined by Henry's Law as:

$$
c_{s}\left(p_{\mathrm{O}_{2}}\right)=\alpha p_{\mathrm{O}_{2}}
$$

with $\alpha$ being the coefficient of oxygen solubility in plasma. As noted in [15] and [17] the dissolved component of blood oxygen is small, unless under hyperoxic condition.

\subsection{Equations of oxygen transport by blood}

Instead of an equation for total oxygen concentration transport, two differential equations can be written for the oxygen transports by haemoglobin and plasma as follows: 


$$
\begin{gathered}
\frac{\partial c_{\mathrm{HbO}_{2}}}{\partial t}+\nabla \cdot\left(v c_{\mathrm{HbO}_{2}}\right)=\nabla \cdot\left(D_{\mathrm{HbO}_{2}} \nabla c_{\mathrm{HbO}_{2}}\right)-\dot{m}_{d} \\
\alpha \frac{\partial p_{\mathrm{O}_{2}}}{\partial t}+\alpha \nabla \cdot\left(v p_{\mathrm{O}_{2}}\right)=\alpha \nabla \cdot\left(D_{p} \nabla p_{\mathrm{O}_{2}}\right)+\dot{m}_{d}-\dot{m}_{c}
\end{gathered}
$$

where $c_{\mathrm{HbO}_{2}}=4 c_{\mathrm{Hb}} S\left(p_{\mathrm{O}_{2}}\right)$ is the oxygen bound in haemoglobin molecules, $\dot{m}_{d}$ is the oxygen mass flow released to plasma due to the oxygen-haemoglobin dissociation reaction, is $v$ the average flow velocity, $D$ the diffusion coefficient and $\dot{m}_{c}$ is oxygen flux delivered to the surrounding tissue through the vessel wall. Although a unique flow velocity has been assumed for plasma and haemoglobin in this work, their velocities may be different.

The above approach was employed in the work by Vadapalli et al. [22]. The work by Pozrikidis and Farrow [23] took into consideration the effect of the fluid flux through the blood vessel walls, which changes the flow rate and pressure in the vasculature. Distribution of oxygen along a straight blood vessel was obtained using a boundary integral method.

The above-mentioned studies dealt with the complex issue of variations of oxygen concentration and partial pressure along each segment length. In this work, a more simplified model of oxygen transport was developed based on the assumption that dissolved oxygen is low in concentration and does not contribute significantly to the oxygen transport by the blood. Ignoring the diffusion of oxygen along blood vessels the transport eqn. (5) can be written as:

$$
c_{H b}\left(\frac{\partial S}{\partial t}+v \cdot \nabla S\right)+S\left(\frac{\partial c_{H b}}{\partial t}+\nabla \cdot\left(v c_{H b}\right)\right)=-0.25 \dot{m}_{c} .
$$

eqn. (7) is seen to comprise of two transport equations, one for haemoglobin and the other for $\mathrm{S}$, as follows:

$$
\begin{gathered}
\frac{\partial c_{H b}}{\partial t}+\nabla \cdot\left(v c_{H b}\right)=0 \\
c_{H b}\left(\frac{\partial S}{\partial t}+v \cdot \nabla S\right)=-0.25 \dot{m}_{c} .
\end{gathered}
$$

The two transport equations above are solved in sequence as the transport of haemoglobin is decoupled from the transport of $S$. Apart from the transport by blood convection, the concentration of haemoglobin may be altered due to interaction of RBCs with blood vessel geometry at the branching points as described in more details below. With the haemoglobin saturation $S$ correlated with the oxygen partial pressure $p_{\mathrm{O}_{2}}$ as described by eqn. (3), eqn. (9) for $S$ can be converted into an equation for $p_{\mathrm{O}_{2}}$ as follows:

$$
c_{H b}\left(\frac{\partial p_{O_{2}}}{\partial t}+v \cdot \nabla p_{O_{2}}\right)=-0.25 \dot{m}_{c} \frac{p_{O_{2}}\left[1+\left(p_{50} / p_{O_{2}}\right)^{n}\right]^{2}}{n\left(p_{50} / p_{O_{2}}\right)^{n}} .
$$




\subsection{Change of red blood cell concentration at branching points}

At bifurcation points in a microvascular network, separation of the RBCs may occur since the RBC are comparable in size with the blood vessels. Therefore, their interaction with the bifurcation geometry could lead to behaviour different from that of the bulk flow. The experimental study by Pries et al. [24] provides some insight into the RBC separation phenomenon. Most remarkably, the study indicated that the interaction at a bifurcation point would lead to more RBCs going into smaller daughter branches and the separation effect depends strongly on the joining-vessel diameters, but not on bifurcation angle. At a bifurcation point shown in Fig. 2(a), the conservations of overall flow and oxygen concentration dictate:

$$
\begin{gathered}
Q_{o}^{\text {out }}=Q_{1}^{\text {in }}+Q_{2}^{\text {in }}, \\
Q_{o}^{\text {out }} c_{o}^{\text {out }}=Q_{1}^{\text {in }} c_{1}^{\text {in }}+Q_{2}^{\text {in }} c_{2}^{\text {in }} .
\end{gathered}
$$

Using eqn. (11) and eqn. (12), as well as RBC separation at the bifurcation point described above, the following relationship can be obtained:

$$
Q_{1}^{\text {in }}\left(1-\frac{c_{1}^{\text {in }}}{c_{o}^{\text {out }}}\right)=-Q_{2}^{\text {in }}\left(1-\frac{c_{2}^{\text {in }}}{c_{o}^{\text {out }}}\right) .
$$

\subsection{Oxygen transport inside the vascular network and in the surrounding tissues}

With only one transport equation for haemoglobin oxygen, the dynamics of oxyhaemoglobin dissociation reaction can not be taken into account. Therefore, a balance of the oxygen dissociated from haemoglobin and that diffused into and consumed by the tissue has been assumed. Diffusion of oxygen across the blood vessel wall can simply be defined by Fick's Law:

$$
\dot{m}_{c}=D_{w}\left(p_{O_{2}}-p_{O 2}^{\text {tis }}\right)\left(A_{w} / V\right),
$$

where $D_{w}$ and $A_{w}$ are the oxygen diffusivity and area of the wall, and $p_{\mathrm{O}_{2}}^{\text {tis }}$ is the tissue oxygen partial pressure. The oxygen distribution inside tissue is described by a diffusion equation as follows:

$$
\frac{\partial c_{O_{2}}^{t i s}}{\partial t}=\nabla \cdot\left(D_{t i s} \nabla c_{O_{2}}^{t i s}\right)+\dot{m}_{c}-\dot{m}_{M R O_{2}},
$$

where $D_{\text {tis }}$ indicates the oxygen diffusivity of tissue and $\dot{m}_{\mathrm{MRO}_{2}}$ denotes the metabolic (consumption) rate of oxygen by the tissue.

Without the oxygen transport by haemoglobin, transport of oxygen dissolved in the tissue is described by an equation similar to eqn. (4) and the equation of oxygen diffusion becomes:

$$
\alpha_{t i s} \frac{\partial p_{O_{2}}^{t i s}}{\partial t}=\alpha_{t i s} \nabla \cdot\left(D_{t i s} \nabla p_{O_{2}}^{t i s}\right)+\dot{m}_{c}-\dot{m}_{M R O_{2}}
$$


In a number of modelling investigations [3, 25], a common oxygen partial pressure was assumed for all the tissue volume under consideration. As a result, the issue of oxygen diffusion in the tissue became irrelevant. In some other studies [2, 15, 17, 18], three-dimensional distribution of oxygen was considered and the models of oxygen transport in the blood and the tissue were coupled using a Green's function method.

\section{Results and discussion}

\subsection{Effect of modelling species transport with pulsatile flow}

Predictions of species transport in the MCA branching model by steady-state and pulsatile flows are shown in Fig. 1. In these simulations, the initial and boundary conditions: $c(\mathrm{t}=0)=0$ and $c($ root $)=0.1$, were applied. Significant difference in the transients of the concentration at different branching levels was observed, especially when the blood viscosity was assumed to be constant. The smaller difference between the transport by steady-state and pulsatile flows, when the blood viscosity was dependent on the blood vessel size was probably a result of increasing blood flow rate in the whole vascular system (see [19]).

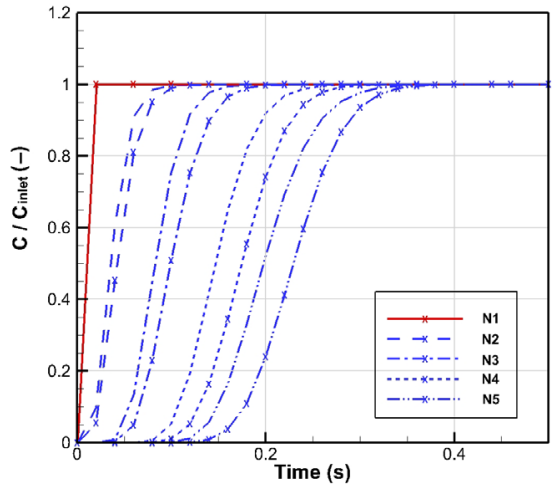

(a)

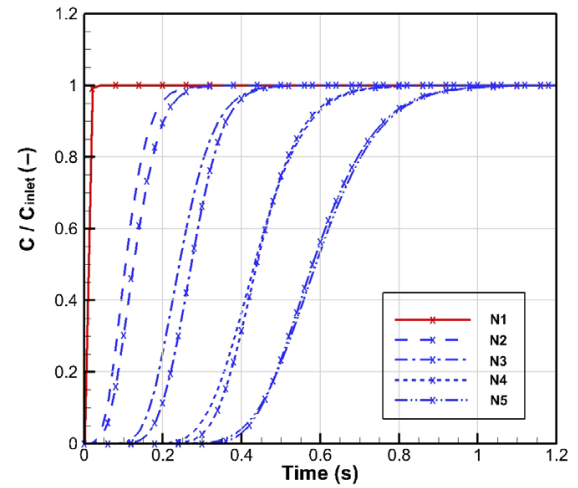

(b)

Figure 1: Transport of a species in the MCA branching tree model by steadystate and pulsatile flows: (a) size dependent vessel compliance, and (b) size dependent vessel compliance and blood viscosity. Refer to Table 1 for N1, N2, N3, N4 and N5. In the figures the graphs with increasing node $(\mathrm{N})$ are left to right. The lines with symbols are for pulsatile flow.

\subsection{Effect of modelling RBC separation at bifurcation points}

The effect of RBC separation at bifurcation points was investigated by introducing 5\% higher RBC concentration of the blood flowing into smaller branches (i.e., $C_{\text {in }}^{\text {branch }} / C_{\text {out }}^{\text {common }}=1.05$ ). The prediction results with RBC separation 
are displayed by lines with symbols in Fig. 2(b). Significant deviations of RBC concentration (up to 15\%) from the corresponding case without RBC separation (lines without symbols) are observed for nodes at different levels of the MCA branching tree model. The deviations are also seen to amplify in the successive levels of the branching tree model, and in certain flow paths lead to the increase of RBC concentration as seen at point N3. Therefore, the branching tree geometry can be an important factor influencing the transport and distribution of RBCs (and dispersed species whose sizes are comparable to the blood vessel sizes) in the branching network of small blood vessels. Instead of a deterministic model of material-bifurcation interaction which can be derived from the work by Pries et al. [24], a statistical interaction model may also be used as in the work by Chung et al. [4]. Such a model can provide an insight into the stochatic nature of such a material-bifurcation interaction and more realistic description of the material transport in vascular branching networks.

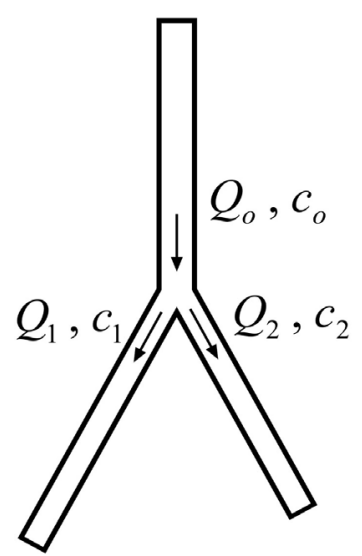

(a)

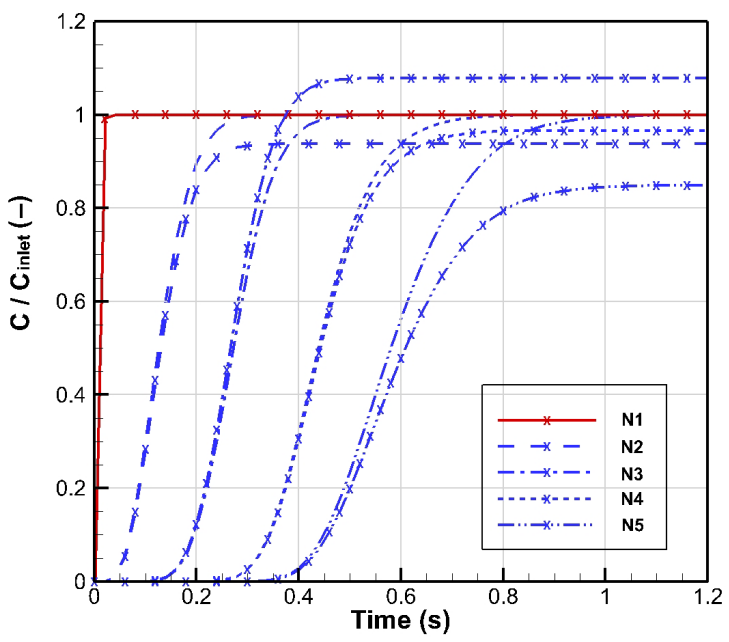

(b)

Figure 2: (a) Flow and oxygen concentration at a bifurcation point. (b) Transport of RBCs in the MCA branching tree model by pulsatile flow with separation of RBCs at branching points. The corresponding simulation results without RBC separation are shown by lines without symbols.

\subsection{Effect of oxygen diffusion across the blood vessel wall}

The transport of oxygen in the MCA network was investigated with the oxygen partial pressure of the tissue assumed to be constant. As described earlier, a 5\% increase of the RBC (and haemoglobin) concentration of the blood flowing into smaller branches was introduced to imitate the effect of blood cell separation at the branching points. With the discharge hematocrit assumed to be $45 \%$ the initial and inlet haemoglobin concentration was set equal $15.75 \%$ by volume. 
Fig. 3 shows the distribution of $p_{\mathrm{O}_{2}}$ in a small MCA branching vascular model without (left) and with (right) oxygen diffusion across the blood vessel wall at time 2s. When oxygen diffusion across the blood vessel wall is modelled the oxygen concentration within the arterial tree varies and is more realistic.
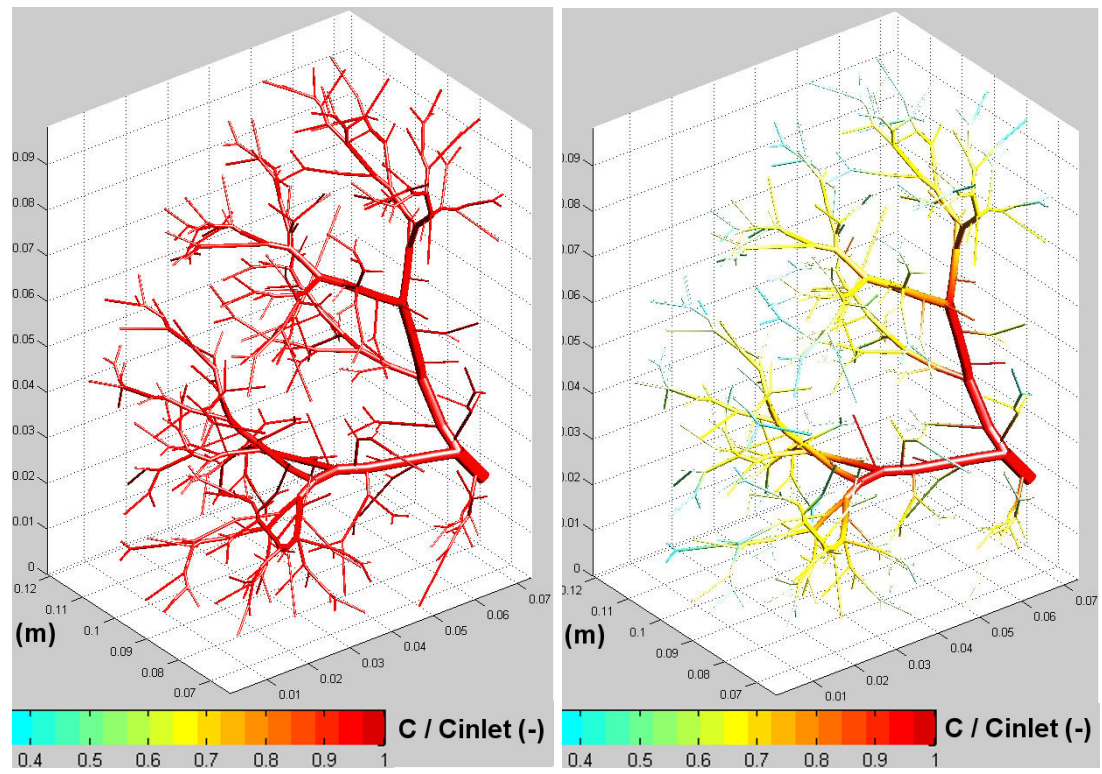

Figure 3: Distribution of $p_{\mathrm{O}_{2}}$ in a small MCA branching vascular model without (left) and with (right) oxygen diffusion across the blood vessel wall at time 2s.

\subsection{Effect of modelling RBC-bifurcation on total oxygen content}

The predictions of total oxygen content for the cases without and with RBCbifurcation interaction are presented in Fig. 4. Without the effect of RBC separation at branching points, gradual decrease of oxygen concentration in the successive branch levels is observed which is induced by the outward oxygen diffusion into the tissue. The separation of RBCs at branching points has a profound effect on the haemoglobin transport and, consequently, oxygen distribution in the vascular branching network as seen in Fig. 4 (right). The increase of RBC concentration along certain flow paths (as explained in section 3.2) is seen to cause the increase of oxygen concentration at point N3 above the inlet concentration. At higher branching tree levels (e.g., N5) the oxygen concentration is lower when RBC separation is included.

\subsection{Current model and future model improvements}

In this work, a model of dynamic material transport was developed and coupled with the model of pulsatile flow in the fractal network of the cerebral 

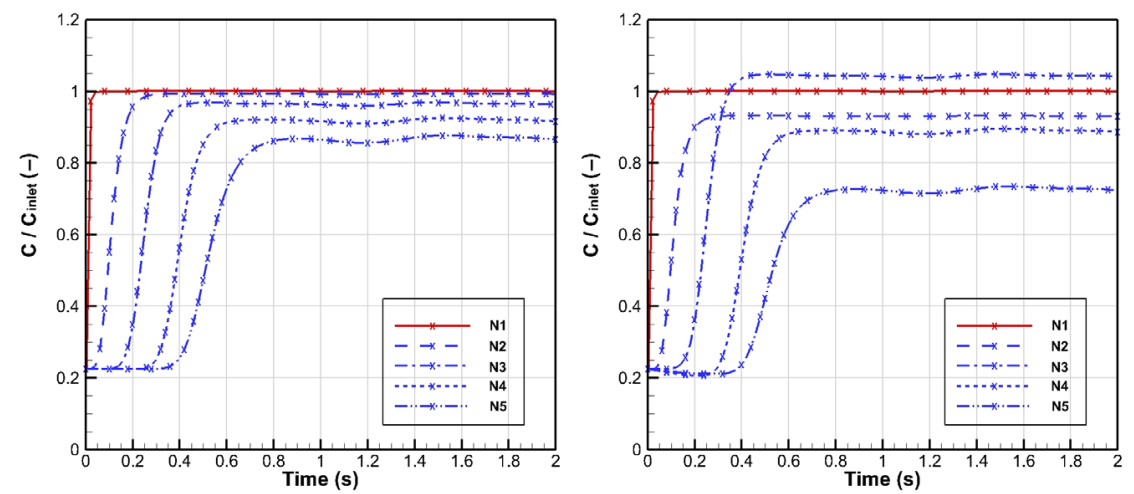

Figure 4: Distribution of oxygen in the MCA branching tree model by pulsatile flow without (left) and with (right) RBC separation at branching points.

microvasculature. The model included blood descriptions of vessel compliance, variable blood rheology, material-geometry interaction, effect of RBC separation at bifurcation points and diffusion across the blood vessel wall. The predicted results indicate the significance of the vessel geometry and RBC-bifurcation point interaction in defining the blood flow and oxygenation by the fractal vasculature. The model can assist in developing a better understanding of into oxygenation in the brain.

This model of the transport of materials in the complex multi-branching structure of the cerebral vasculature could be used to predict the brain oxygenation under abnormal conditions, when changes in cerebral flow result from aging, diseases, and metabolism. For example, the effect of blood vessel wall vasoconstriction (narrowing), wall structural property (elasticity) or inlet flow on cerebral blood flow and brain oxygenation.

Future extension of this transport model could include coupling with a model of the three-dimensional oxygen transport and consumption inside the cerebral tissue, so that the effect of local variation of metabolism rate and hypoxia can be predicted. The model can also be improved by including a more accurate geometry representation of the preferential distribution of large arteries on the brain cortex as per Bui et al. [26]. The posterior and anterior cerebral vasculature which have certain variations in structure and geometry compared to the MCA will be investigated in a future study.

\section{Conclusion}

A mathematical model of material transport in a branching tree network of the MCA cerebral vasculature was developed which incorporated transient flow, full network structure, material-geometry interaction and diffusion across the blood vessel wall. 
The model showed that transport of species in the branching tree differs between transient flow and steady flow. The branching tree geometry can be an important factor in influencing the transport and distribution of RBCs. The RBC separation at branching points had a large affect on the oxygen distribution in the vascular branching network. It is shown that oxygen diffusion across the wall needs to be modelled to better simulate the oxygen distribution.

In future, this model can be used to predict the brain oxygenation in the complex multi-branching structure of the cerebral vasculature under diseased conditions with changed cerebral flow.

\section{References}

[1] Macheras, P., Iliadis, A., Modeling in biopharmaceutics, pharmacokinetics, and pharmacodynamics - Homogeneous and Heterogeneous approaches. Springer, 2006.

[2] Secomb, T., Hsu, R., Beamer, N. and Coull, B., Theoretical simulation of Oxygen transport to brain by networks of microvessels: Effects of Oxygen supply and demand on tissue hypoxia. Microcirc, 7, pp. 237-247, 2000.

[3] Boas, D.A., Jones, S.R., Devor, A., Huppert, T.J. and Dale, A.M., A vascular anatomical network model of the spatio-temporal response to brain activation. NeuroImage, 40, pp. 1116-1129, 2008.

[4] Chung, E.M.L., Hague, J.P. and Evans, D.H., Revealing the mechanisms underlying embolic stroke using computational modelling. Phys Med Biol 52, pp. 7153-7166, 2007.

[5] Šutalo, I.D., Bui, A., Ahmed, S., Liffman, K. and Manasseh, R., Modelling of embolus transport and embolic stroke, Environmental Health and Biomedicine, WIT Transactions on Biomedicine and Health, Vol. 15., BioMED 2011 (9th International Conference in Medicine and Biology), Riga, Latvia, pp. 347-358, 2011.

[6] Olufsen, M.S., Peskin, C.S., Kim, W.Y., Pedersen, E.M., Nadim, A. and Larsen, J., Numerical simulation and experimental validation of blood flow in arteries with structured-tree outflow conditions. Annals of Biomed Eng, 28, pp. 1281-1299, 2000.

[7] Olufsen, M.S., Structured tree outflow condition for blood flow in larger systemic arteries. Am J Physiol, 276, pp. H257-268, 1999.

[8] Gabryś, E., Rybaczuk, M. and Kędzia, A., Blood flow simulation through fractal models of circulatory system. Chaos, Solitons and Fractals, 27, pp. 1-7, 2006.

[9] Mayer, S., On the pressure and flow-rate distributions in tree-like and arterial-venous networks. Bull Math Biology, 58, pp. 753-785, 1996.

[10] Sharma, H.S., Westman, J. (eds.). Blood-spinal cord and brain barriers in health and disease. Elsevier Academic Press, 2004.

[11] Edvinsson, L., Mackenzie, E.T. and McCulloch, J., Cerebral blood flow and metabolism. Raven Press, 1993. 
[12] Beard, D.A. and Bassingthwaighte, J.B., Advection and diffusion of substances in biological tissues with complex vascular networks. Annals of Biomed Eng, 28, pp. 253-268, 2000.

[13] Dokoumetzidis, A. and Macheras, P., A model for transport and dispersion in the circulatory system based on the vascular fractal tree. Annals of Biomed Eng, 31, pp. 284-293, 2003.

[14] Karshafian, R., Burns, P.N. and Henkelman, M., Transit time kinetics in ordered and disordered vascular trees. Phys Med Biol, 48, pp. 3225-3237, 2003.

[15] Kavanagh, B., Secomb, T., Hsu, R., Lin, P., Venitz, J. and Dewhirst, M., A theoretical model for the effects of reduced Hemoglobin-Oxygen affinity on tumor oxygenation. Int J Radiat Oncol, 53, pp. 172-179, 2002.

[16] Macheras, A.D.P., A model for transport and dispersion in the circulatory system based on the vascular fractal tree. Annals Biomed Eng, 31, pp. 284293, 2003.

[17] Secomb, T., Hsu, R., Park, E. and Dewhirst, M., Green's function methods for analysis of Oxygen delivery to tissue by microvascular networks. Ann Biomed Eng, 32, pp. 1519-1529, 2004.

[18] Tsoukias, N., Goldman, D., Vadapalli, A., Pittman, R. and Popel, A., A computational model of oxygen delivery by hemoglobin-based oxygen carriers in three-dimensional microvascular networks. J Theor Biol, 248, pp. 657-674, 2007.

[19] Bui, A., Šutalo, I.D., Manasseh, R. and Liffman, K., Dynamics of pulsatile flow in fractal models of vascular branching networks. Med Biol Eng Comput, 47, pp. 763-772, 2009.

[20] Šutalo, I.D., Bui, A., Ahmed, S., Liffman, K. and Manasseh, R., Modelling of flow through the circle of Willis and cerebral vasculature, Modelling in Medicine and Biology VIII, WIT Transactions on Biomedicine and Health, Vol. 13., BioMED 2009, Crete, Greece, pp. 83-92, 2009.

[21] Schreiner, W., Karch, R., Neumann, M., Neumann, F., Szawlowski, P. and Roedler, S., Optimized arterial trees supplying hollow organs. Med Eng and Phys, 28, pp. 416-429, 2006.

[22] Vadapalli, A., Goldman, D. and Popel, A., Calculations of oxygen transport by red blood cells and hemoglobin solutions in capillaries. Artif Cell Blood Sub, 30(3), pp. 157-188, 2002.

[23] Pozrikidis, C. and Farrow, D., A model of fluid flow in solid tumors. Ann Biomed Eng, 31, pp. 181-194, 2003.

[24] Pries, A.R., Leg, K., Claassen, M. and Gaehtgens, P., Red cell distribution at microvascular bifurcations. Microvasc Res, 38, pp. 81-101, 1989.

[25] Sharan, M. and Popel, A., A compartmental model for oxygen transport in brain microcirculation in the presence of blood substitutes. $J$ Theor Boil, 216, pp. 479-500, 2002.

[26] Bui, A.V., Manasseh, R., Liffman, K. and Šutalo, I.D., Development of optimized vascular fractal tree models using level set distance function. Med Eng and Phys, 32, pp. 790-794, 2010. 